\title{
AN ITERATED LOGATITHM RESULT FOR MARTIN- GALES AND ITS APPLICATION IN ESTIMATION THEORY FOR AUTOREGRESSIVE PROCESSES
}

BY

C. C. HEYDE

Reprinted from the JOURNAL OF APPLIED PROBABILITY

Vol. 10, No. 1, pp. 146-157

March 1973 


\title{
AN ITERATED LOGARITHM RESULT FOR MARTINGALES AND ITS APPLICATION IN ESTIMATION THEORY FOR AUTOREGRESSIVE PROCESSES
}

\author{
C. C. HEYDE, The Australian National University
}

\begin{abstract}
The paper begins with an iterated logarithm law of classical HartmanWintner form for stationary martingales. This is then used to obtain iterated logarithm results giving information on rates of convergence of estimators of the parameters in a stationary autoregressive process. In the case of an autoregression of small order, detailed rate results for each autocorrelation and for the estimators of all parameters can be obtained. A rate result for the convergence of the sample mean is given in the general case.

ITERATED LOGARITHM LAW; MARTINGALES; TIME SERIES ESTIMATION; AUTOREGRESSIVE PROCESS
\end{abstract}

\section{Results and discussion}

In this paper we begin by obtaining the following iterated logarithm law for martingales.

Theorem 1. Let $\left\{Y_{n}, n \geqq 0\right\}$ be a stationary stochastic sequence such that $E\left(Y_{n} \mid \mathscr{G}_{n-1}\right)=0$ a.s., all $n \geqq 1$, and $E Y_{0}^{2}=1$. Here $\mathscr{G}_{n}$ is the $\sigma$-field generated by $Z_{i}, i \leqq n$, where $\left\{\cdots, Z_{-1}, Z_{0}, Z_{1}, \cdots\right\}$ is a stationary stochastic sequence such that $Y_{k}=\phi\left(Z_{k}, Z_{k-1}, \cdots\right), k \geqq 0$, for some measurable $\phi$. We suppose also that $n^{-1} \sum_{i=1}^{n} E\left(Y_{i}^{2} \mid \mathscr{G}_{i-1}\right)$ converges almost surely (a.s.) to 1 as $n \rightarrow \infty$. Then,

$$
\begin{aligned}
& \underset{n \rightarrow \infty}{\limsup } \sum_{i=1}^{n} Y_{i} /(2 n \log \log n)^{\frac{1}{2}}=1 \text { a.s., } \\
& \liminf _{n \rightarrow \infty} \sum_{i=1}^{n} Y_{i} /(2 n \log \log n)^{\frac{1}{2}}=-1 \text { a.s. }
\end{aligned}
$$

This result is an extension of that of Stout [3] who has taken $Z_{i}=Y_{i}$ and assumed that $\left\{Y_{i}\right\}$ is a stationary ergodic martingale difference sequence $\left(n^{-1} \sum_{i=1}^{n} E\left(Y_{i}^{2} \mid \mathscr{G}_{i-1}\right) \stackrel{\text { a.s. }}{\rightarrow} 1\right.$ then follows as a consequence of the ergodic theorem). Our result seems more useful for applications; in particular the essentially uncheckable ergodicity assumption is replaced by something rather more concrete.

Received 13 December 1971. 
The point of introducing the $Z$ 's will be clear from some of our applications where a conditioning on the $\sigma$-fields generated by the $Y$ 's themselves is not at all convenient.

We go on to apply Theorem 1 in a time series context. We consider a stationary autoregression $\{x(n)\}$ of the form

$$
\begin{aligned}
& \sum_{k=0}^{q} \beta(k)\{x(n-k)-\mu\}=\varepsilon(n) ; \quad \beta(0)=1 ; \\
& E \varepsilon(n)=0, E\{\varepsilon(m) \varepsilon(n)\}=0, \quad m \neq n,
\end{aligned}
$$

where we assume that

$$
\sum_{k=0}^{q} \beta(k) z^{k} \neq 0, \quad|z| \leqq 1
$$

We shall also suppose that $\{\varepsilon(n)\}$ is a stationary stochastic sequence with $E \varepsilon^{2}(1)=\sigma^{2}$ satisfying

$$
E\left(\varepsilon(n) \mid \mathscr{F}_{n-1}\right)=0 \text { a.s., all } n,
$$

where $\mathscr{F}_{n}$ is the $\sigma$-field generated by $\varepsilon(m), m \leqq n$ and

$$
E\left(\varepsilon^{2}(n) \mid \mathscr{F}_{n-1}\right)=\sigma^{2} \text { a.s., all } n \text {. }
$$

In this context, $\mathscr{F}_{n}$ is also the $\sigma$-field generated by $x(m), m \leqq n$, and

$$
\varepsilon(n)=x(n)-E\left(x(n) \mid \mathscr{F}_{n-1}\right)
$$

so that (3) is equivalent to assuming that $E\left(x(n) \mid \mathscr{F}_{n-1}\right)$ is the best linear predictor and the best linear predictor is the best predictor (both in the least squares sense). We are also assuming that $E\left(\varepsilon^{2}(n) \mid \mathscr{F}_{n-1}\right)$, the prediction variance, given the past, is a.s. constant.

Let $x(1), x(2), \cdots, x(N)$ be a sample of $N$ consecutive observations on the process $\{x(n)\}$ and define the autocovariances

$$
\begin{aligned}
& c(j) \quad=N^{-1} \sum_{n=1}^{N-j}(x(n)-\bar{x})(x(n+j)-\bar{x}), \quad j \geqq 0, \\
& c(-j)=c(j),
\end{aligned}
$$

where $\bar{x}=N^{-1} \sum_{j=1}^{N} x(j)$. It follows from more general results given in Hannan and Heyde [2] that the $\beta(j)$ and $\sigma^{2}$ can be estimated through

$$
\sum_{j=0}^{q} \hat{\beta}(j) c(k-j)=\delta_{0 k} \hat{\sigma}^{2}, \quad k=0,1, \cdots, q,
$$

where $\delta_{0 k}$ is the Kronecker delta, while $\mu$ can be estimated by $\bar{x}$ (see also Hannan [1], Chapter VI). In fact, 


$$
\lim _{N \rightarrow \infty} \sum_{j=0}^{q} \beta(j) c(k-j)=\delta_{0 k} \sigma^{2} \text { a.s., } \quad k=0,1, \cdots, q,
$$

and if $r(j)=c(j) / c(0)$ are the autocorrelations,

$$
\lim _{N \rightarrow \infty} \sum_{j=0}^{q} \beta(j) r(k-j)=0 \text { a.s., } \quad k=1,2, \cdots, q .
$$

Also,

$$
\lim _{N \rightarrow \infty} \bar{x}=\mu \text { a.s. }
$$

The results of [2] extend the classical theory in which the $\varepsilon(n)$ are assumed to be independent and identically distributed (i.i.d.).

We shall here obtain iterated logarithm results which illuminate the Relations (5), (6) and (7) in the sense of providing detailed information on the rate of a.s. convergence. The results are new even under the additional assumption that the $\varepsilon(n)$ are i.i.d. First we deal with (6) in the following theorem.

Theorem 2. Suppose $E \varepsilon^{4}(n)<\infty$. For any $k, 1 \leqq k \leqq q$, we have

$$
\begin{aligned}
& \underset{N \rightarrow \infty}{\limsup } N^{\frac{1}{2}}(2 \log \log N)^{-\frac{1}{2}} \sum_{j=0}^{q} \beta(j) r(k-j)=c^{-\frac{1}{2}} \text { a.s., } \\
& \underset{N \rightarrow \infty}{\liminf } N^{\frac{1}{2}}(2 \log \log N)^{-\frac{1}{2}} \sum_{j=0}^{q} \beta(j) r(k-j)=-c^{-\frac{1}{2}} \text { a.s., }
\end{aligned}
$$

where $c=\sum_{j=0}^{\infty} \alpha^{2}(j)$, the $\alpha$ 's being defined by $\sum_{j=0}^{q} \beta(j) \alpha(n-j)=\delta_{0 n}, \alpha(k)=0$, $k<0$. Thus, for $1 \leqq k \leqq q$,

$$
\sum_{j=0}^{n} \beta(j) r(k-j)=\zeta_{k}(N) c^{-\frac{1}{2}} N^{-\frac{1}{2}}(2 \log \log N)^{\frac{1}{2}},
$$

where $\zeta_{k}(N)$ has its set of limit points confined to $[-1,1]$ with $\lim \sup _{N \rightarrow \infty} \zeta_{k}(N)=1$ a.s. and $\liminf _{N \rightarrow \infty} \zeta_{k}(N)=-1$ a.s.

Remark 1. It should be noted that, using Theorem 2 of [2], $N^{\frac{1}{2}} \sum_{j=0}^{q} \beta(j) r(k-j)$ converges in distribution as $N \rightarrow \infty$ to a normal law with zero mean and specified variance $v(k), 1 \leqq k \leqq q$.

Remark 2. In the case of a first order autoregression $(q=1)$, Theorem 2 gives

$$
r(1)=\rho+\zeta_{1}(N)\left(1-\rho^{2}\right)^{\frac{1}{2}} N^{-\frac{1}{2}}(2 \log \log N)^{\frac{1}{2}},
$$

where $\beta(1)=-\rho$ since in this case $\alpha(n)=\rho^{n}, n \geqq 0$. For $q \geqq 2$, Theorem 2 does not generally give such precise results on the individual autocorrelations. For example, for $q=2$ we find 


$$
r(1)=-\frac{\beta(1)}{1+\beta(2)}+\zeta_{1}(N) \frac{c^{-\frac{1}{2}}}{1+\beta(2)} N^{-\frac{1}{2}}(2 \log \log N)^{\frac{1}{2}}
$$

However, the best we can do for $r(2)$ is

where

$$
r(2)=\frac{\beta^{2}(1)}{1+\beta(2)}-\beta(2)+\eta(N) c^{-\frac{1}{2}} N^{-\frac{1}{2}}(2 \log \log N)^{\frac{1}{2}},
$$

and

$$
\limsup _{N \rightarrow \infty} \eta(N) \leqq 1+\left|\frac{\beta(1)}{1+\beta(2)}\right| \text { a.s. }
$$

$$
\liminf _{N \rightarrow \infty} \eta(N) \geqq-1-\left|\frac{\beta(1)}{1+\beta(2)}\right| \text { a.s. }
$$

Remark 3. Since the $\hat{\beta}$ 's are linear combinations of the $r$ 's we can, at least for small $q$, also use Theorem 2 to find convenient rate results for the convergence of $\hat{\beta}(j)$ to $\beta(j), 1 \leqq j \leqq q$.

In Theorem 2 we have dealt with the System (6). As a by-product of the proof we shall obtain for $1 \leqq k \leqq q$,

$$
\begin{aligned}
& \underset{N \rightarrow \infty}{\limsup } N^{\frac{1}{2}}(2 \log \log N)^{-\frac{1}{2}} \sum_{j=0}^{q} \beta(j) c(k-j)=\sigma^{2} c^{\frac{1}{2}} \text { a.s. } \\
& \underset{N \rightarrow \infty}{\liminf } N^{\frac{1}{2}}(2 \log \log N)^{-\frac{1}{2}} \sum_{j=0}^{q} \beta(j) c(k-j)=-\sigma^{2} c^{\frac{1}{2}} \text { a.s., }
\end{aligned}
$$

(see (23)) which deals with the System (5) save for the case $k=0$. This case we treat in the next theorem. Here we find it convenient, in order to avoid unpleasant extra assumptions, to specialize to the case where the $\varepsilon(n)$ are independent.

Theorem 3. Suppose that in addition to our basic assumptions the $\varepsilon(n)$ are independent with $E \varepsilon^{4}(n)=\mu_{4}<\infty$. Then,

$$
\sum_{j=0}^{q} \beta(j) c(j)=\sigma^{2}+\left[\mu_{4}+\sigma^{4}(c-2)\right]^{\frac{1}{2}} \eta(N) N^{-\frac{1}{2}}(2 \log \log N)^{\frac{1}{2}},
$$

where $\eta(N)$ has its set of limit points confined to $[-1,1]$ with $\lim \sup _{N \rightarrow \infty} \eta(N)=1$ a.s. and $\lim \inf _{N \rightarrow \infty} \eta(N)=-1$ a.s.

Finally, we deal with (7) in the following theorem.

Theorem 4. We have

$$
\bar{x}=\mu+\sigma\left(\sum_{k=0}^{q} \beta(k)\right)^{-1} \delta(N) N^{-\frac{1}{2}}(2 \log \log N)^{\frac{1}{2}},
$$

where $\delta(N)$ has its set of limit points confined to $[-1,1]$ with lim $\sup _{N \rightarrow \infty} \delta(N)=1$ a.s. and $\liminf _{N \rightarrow \infty} \delta(N)=-1$ a.s. 


\section{Proofs}

We require first the following lemma which provides a strong law for stationary martingales.

Lemma. Let $\left\{U_{n}, n \geqq 0\right\}$ be a stationary sequence of random variables and $\left\{\mathscr{G}_{n}, n \geqq 0\right\}$ an increasing sequence of $\sigma$-fields such that $U_{n}$ is measurable with respect to $\mathscr{G}_{n}$ and $\left\{E\left(U_{n} \mid \mathscr{G}_{n-1}\right), n \geqq 1\right\}$ is a stationary sequence. If $E\left|U_{n}\right|<\infty$, then

$$
n^{-1} \sum_{k=1}^{n}\left[U_{k}-E\left(U_{k} \mid \mathscr{G}_{k-1}\right)\right] \stackrel{\text { a.s. }}{\rightarrow} 0
$$

as $n \rightarrow \infty$.

Proof. First we show that

$$
n^{-1} \sum_{k=1}^{n}\left[U_{k}-E\left(U_{k} \mid \mathscr{G}_{k-1}\right)\right] \stackrel{p}{\rightarrow} 0
$$

as $n \rightarrow \infty$ (" $p$ " denotes convergence in probability). Let $U_{k n}=U_{k}$ or 0 according as $\left|U_{k}\right| \leqq n$ or $\left|U_{k}\right|>n$. Then

$$
\begin{gathered}
P\left(n^{-1} \sum_{k=1}^{n}\left[U_{k}-E\left(U_{k} \mid \mathscr{G}_{k-1}\right)\right] \neq n^{-1} \sum_{k=1}^{n}\left[U_{k n}-E\left(U_{k} \mid \mathscr{G}_{k-1}\right)\right]\right) \\
\leqq \sum_{k=1}^{n} P\left(U_{k} \neq U_{k n}\right)=n P\left(\left|U_{k}\right|>n\right) \rightarrow 0
\end{gathered}
$$

as $n \rightarrow \infty$ since $E\left|U_{k}\right|<\infty$. Furthermore,

$$
\begin{aligned}
& E\left|n^{-1} \sum_{k=1}^{n}\left\{E\left(U_{k} \mid \mathscr{G}_{k-1}\right)-E\left(U_{k n} \mid \mathscr{G}_{k-1}\right)\right\}\right| \\
& \leqq n^{-1} \sum_{k=1}^{n} E\left\{E\left(\left|U_{k}\right| I\left(\left|U_{k}\right|>n\right) \mid \mathscr{G}_{k-1}\right)\right\} \\
&=E\left(\left|U_{k}\right| I\left(\left|U_{k}\right|>n\right)\right) \rightarrow 0
\end{aligned}
$$

as $n \rightarrow \infty$ since $E\left|U_{k}\right|<\infty$ ( $I$ denotes the indicator function). We then conclude, using Markov's inequality, that

$$
n^{-1} \sum_{k=1}^{n}\left\{E\left(U_{k} \mid \mathscr{G}_{k-1}\right)-E\left(U_{k n} \mid \mathscr{G}_{k-1}\right)\right\} \stackrel{p}{\rightarrow} 0 .
$$

Next, using Chebyshev's inequality and the orthogonal increments property of the martingale, 


$$
\begin{aligned}
P\left(n^{-1} \mid \sum_{k=1}^{n}\left[U_{k n}-\right.\right. & \left.\left.E\left(U_{k n} \mid \mathscr{G}_{k-1}\right)\right] \mid>\varepsilon\right) \\
& \leqq n^{-2} \varepsilon^{-2} \sum_{k=1}^{n} E\left[U_{k n}-E\left(U_{k n} \mid \mathscr{G}_{k-1}\right)\right]^{2} \\
& \leqq n^{-1} \varepsilon^{-2} E\left(U_{k n}\right)^{2} \\
& \leqq 2 n^{-1} \varepsilon^{-2} \int_{0}^{n} x P\left(\left|U_{k}\right|>x\right) d x \rightarrow 0
\end{aligned}
$$

as $n \rightarrow \infty$ since $x P\left(\left|U_{k}\right|>x\right) \rightarrow 0$ as $x \rightarrow \infty$ by virtue of $E\left|U_{k}\right|<\infty$. Thus, from (9), (10) and (11) we obtain (8).

We now make two applications of the ergodic theorem and obtain

$$
\begin{gathered}
n^{-1} \sum_{k=1}^{n} U_{k} \stackrel{\text { a.s. }}{\rightarrow} E\left(U_{1} \mid \mathscr{G}\right), \\
n^{-1} \sum_{k=1}^{n} E\left(U_{k} \mid \mathscr{G}_{k-1}\right) \stackrel{\text { a.s. }}{\rightarrow} E\left(E\left(U_{1} \mid \mathscr{G}_{0}\right) \mid \mathscr{H}\right),
\end{gathered}
$$

where $\mathscr{G}, \mathscr{H}$ are the respective $\sigma$-fields of invariant events. Thus,

$$
n^{-1} \sum_{k=1}^{n}\left[U_{k}-E\left(U_{k} \mid \mathscr{G}_{k-1}\right)\right] \stackrel{\text { a.s. }}{\rightarrow} E\left(U_{1} \mid \mathscr{G}\right)-E\left(E\left(U_{1} \mid \mathscr{G}_{0}\right) \mid \mathscr{H}\right),
$$

and the result of the lemma follows upon identifying in (8) and (12).

Proof of Theorem 1. We retain the notation of [3] but replace $\mathscr{F}_{i}$ by our $\mathscr{G}_{i}\left(\supseteq \mathscr{F}_{i}\right)$. All that we need to do is to show that $n^{-1} \sum_{i=1}^{n} E\left[\left(Z_{i}^{\prime}\right)^{2} \mid \mathscr{G}_{i-1}\right] \stackrel{\text { a.s. }}{\rightarrow} 1$ continues to hold and then the remainder of the proof follows as in [3].

Now suppose that $Y_{0}(\omega)$ is a random variable on $(\Omega, \mathscr{F}, P)$ and let $T$ be the measure preserving transformation of $\Omega$ onto itself such that $Y_{n}(\omega)=Y_{0}\left(T^{n} \omega\right)$. Then,

$$
\begin{aligned}
Y_{0}^{\prime \prime}\left(T^{n} \omega\right) & =Y_{0}\left(T^{n} \omega\right) I\left(\left|Y_{0}\left(T^{n} \omega\right)\right| \leqq M\right)+M I\left(Y_{0}\left(T^{n} \omega\right)>M\right)-M I\left(Y_{0}\left(T^{n} \omega\right)<-M\right) \\
& =Y_{n}(\omega) I\left(\left|Y_{n}(\omega)\right| \leqq M\right)+M I\left(Y_{n}(\omega)>M\right)-M I\left(Y_{n}(\omega)<-M\right) \\
& =Y_{n}^{\prime \prime}(\omega),
\end{aligned}
$$

and from the ergodic theorem

$$
\begin{aligned}
\lim _{n \rightarrow \infty} n^{-1} \sum_{i=1}^{n}\left(Y_{0}^{\prime \prime}\left(T^{i} \omega\right)\right)^{2} & =\lim _{n \rightarrow \infty} n^{-1} \sum_{i=1}^{n}\left(Y_{i}^{\prime \prime}(\omega)\right)^{2} \\
& =E\left[\left(Y_{0}^{\prime \prime}\right)^{2} \mid \mathscr{H}\right] \text { a.s., }
\end{aligned}
$$

where $\mathscr{H}$ is the $\sigma$-field of invariant events determined by $T, \mathscr{F}$. Also, in view of our stationarity assumptions we can apply the lemma to give 


$$
n^{-1} \sum_{i=1}^{n}\left\{\left(Y_{i}^{\prime \prime}\right)^{2}-E\left[\left(Y_{i}^{\prime \prime}\right)^{2} \mid \mathscr{G}_{i-1}\right]\right\} \stackrel{\text { a.s. }}{\rightarrow} 0
$$

and hence

$$
n^{-1} \sum_{i=1}^{n} E\left[\left(Y_{i}^{\prime \prime}\right)^{2} \mid \mathscr{G}_{i-1}\right] \stackrel{\text { a.s. }}{\rightarrow} E\left[\left(Y_{0}^{\prime \prime}\right)^{2} \mid \mathscr{H}\right] .
$$

Next, we note that for $b_{i} \geqq M,\left|Y_{i}\right| \geqq\left|Y_{i}^{\prime}\right| \geqq\left|Y_{i}^{\prime \prime}\right|$ so that

$$
E\left[Y_{i}^{2} \mid \mathscr{G}_{i-1}\right] \geqq E\left[\left(Y_{i}^{\prime}\right)^{2} \mid \mathscr{G}_{i-1}\right] \geqq E\left[\left(Y_{i}^{\prime \prime}\right)^{2} \mid \mathscr{G}_{i-1}\right] \text { a.s. }
$$

and hence, making use of (13),

$$
\begin{aligned}
1=\lim _{n \rightarrow \infty} n^{-1} \sum_{i=1}^{n} E\left[Y_{i}^{2} \mid \mathscr{G}_{i-1}\right] & \geqq \limsup _{n \rightarrow \infty} n^{-1} \sum_{i=1}^{n} E\left[\left(Y_{i}^{\prime}\right)^{2} \mid \mathscr{G}_{i-1}\right] \\
& \geqq \liminf _{n \rightarrow \infty} n^{-1} \sum_{i=1}^{n} E\left[\left(Y_{i}^{\prime}\right)^{2} \mid \mathscr{G}_{i-1}\right] \\
& \geqq \lim _{n \rightarrow \infty} n^{-1} \sum_{i=1}^{n} E\left[\left(Y_{i}^{\prime \prime}\right)^{2} \mid \mathscr{G}_{i-1}\right] \\
& =E\left[\left(Y_{0}^{\prime \prime}\right)^{2} \mid \mathscr{H}\right] \text { a.s. }
\end{aligned}
$$

Also, as $M \uparrow \infty,\left|Y_{0}^{\prime \prime}\right| \uparrow\left|Y_{0}\right|$ so that $E\left[\left(Y_{0}^{\prime \prime}\right)^{2} \mid \mathscr{H}\right] \stackrel{\text { a.s. }}{\rightarrow} E\left[Y_{0}^{2} \mid \mathscr{H}\right]$.

But, an application of the lemma gives

$$
n^{-1} \sum_{i=1}^{n}\left[Y_{i}^{2}-E\left(Y_{i}^{2} \mid \mathscr{G}_{i-1}\right)\right] \stackrel{\text { a.s. }}{\rightarrow} 0,
$$

while $n^{-1} \sum_{i=1}^{n} E\left(Y_{i}^{2} \mid \mathscr{G}_{i-1}\right) \stackrel{\text { a.s. }}{\rightarrow} 1$ by hypothesis and hence

$$
\lim _{n \rightarrow \infty} n^{-1} \sum_{i=1}^{n} Y_{i}^{2}=E\left[Y_{0}^{2} \mid \mathscr{H}\right]=1 \text { a.s. }
$$

Thus, letting $M \rightarrow \infty$ in (14) gives

$$
n^{-1} \sum_{i=1}^{n} E\left[\left(Y_{i}^{\prime}\right)^{2} \mid \mathscr{G}_{i-1}\right] \stackrel{\text { a.s. }}{\rightarrow} 1 .
$$

Now take $\left\{b_{n}\right\}$ as chosen in [3] and observe that

$$
E\left(Y_{i}^{\prime} \mid \mathscr{G}_{i-1}\right)=-E\left(Y_{i}-Y_{i}^{\prime} \mid \mathscr{G}_{i-1}\right),
$$

while $\left|Y_{i}^{\prime}\right| \leqq b_{i}$ so that

$$
E\left[E\left(Y_{i}^{\prime} \mid \mathscr{G}_{i-1}\right)\right]^{2} \leqq b_{i} E\left|Y_{i}-Y_{i}^{\prime}\right| .
$$

Then, $\Sigma i^{-1}\left[E\left(Y_{i}^{\prime} \mid \mathscr{G}_{i-1}\right)\right]^{2}<\infty$ a.s. since 


$$
\sum i^{-1} E\left[E\left(Y_{i}^{\prime} \mid \mathscr{G}_{i-1}\right)\right]^{2} \leqq \Sigma b_{i} i^{-1} E\left|Y_{i}-Y_{i}^{\prime}\right|=\Sigma K_{i}\left(i \log _{2} i\right)^{-\frac{1}{2}} E\left|Y_{i}-Y_{i}^{\prime}\right|<\infty,
$$

it having been shown in [3] that $\Sigma\left(i \log _{2} i\right)^{-\frac{1}{2}} E\left|Y-Y_{i}^{\prime}\right|<\infty$ while $K_{i} \rightarrow 0$ as $i \rightarrow \infty$. Thus, using the Kronecker lemma,

$$
n^{-1} \sum_{i=1}^{n}\left[E\left(Y_{i}^{\prime} \mid \mathscr{G}_{i-1}\right)\right]^{2} \stackrel{\text { a.s. }}{\rightarrow} 0
$$

and hence, making use of (15),

$$
n^{-1} \sum_{i=1}^{n} E\left[\left(Z_{i}^{\prime}\right)^{2} \mid \mathscr{G}_{i-1}\right]=n^{-1} \sum_{i=1}^{n}\left\{E\left[\left(Y_{i}^{\prime}\right)^{2} \mid \mathscr{G}_{i-1}\right]-\left[E\left(Y_{i}^{\prime} \mid \mathscr{G}_{i-1}\right)\right]^{2}\right\}^{\text {a.s. }} \rightarrow
$$

The remainder of the proof is then completed as in [3].

We shall next prove Theorem 4 as it is convenient to use it in the proof of Theorems 2 and 3.

Proof of Theorem 4. First note that an application of Theorem 1 gives

$$
\limsup _{N \rightarrow \infty}(2 N \log \log N)^{-\frac{1}{2}} \sum_{n=1}^{N} \varepsilon(n)=\sigma \text { a.s., }
$$

with a corresponding result for lim inf.

Now,

$$
\begin{aligned}
\sum_{n=1}^{N} \varepsilon(n) & =\sum_{n=1}^{N} \sum_{k=0}^{q} \beta(k)\{x(n-k)-\mu\} \\
& =\sum_{k=0}^{q} \beta(k)\left\{N \bar{x}-N \mu+\sum_{n=1}^{k} x(n-k)-\sum_{n=N+1}^{N+k} x(n-k)\right\} .
\end{aligned}
$$

Also, $E\left(\sum_{n=1}^{k} x(n-k)\right)^{2}<\infty$ and the $R_{N}=\sum_{n=N+1}^{N+k} x(n-k)$ are identically distributed (as $R$, say) with $E R^{2}<\infty$. Thus,

$$
\begin{aligned}
\sum_{N} P\left(\left|\sum_{n=1}^{k} x(n-k)\right| \geqq \varepsilon(N \log \log N)^{\frac{1}{2}}\right) \\
\leqq \sum_{N} P\left(\left|\sum_{n=1}^{k} x(n-k)\right| \geqq \varepsilon N^{\frac{1}{2}}\right)<\infty,
\end{aligned}
$$

and

$$
\sum_{N} P\left(\left|R_{N}\right| \geqq \varepsilon(N \log \log N)^{\frac{1}{2}}\right) \leqq \sum_{N} P\left(|R| \geqq \varepsilon N^{\frac{1}{2}}\right)<\infty
$$

for any $\varepsilon>0$. Hence, from the Borel-Cantelli lemma,

$$
\left(\sum_{n=1}^{k} x(n-k)-R_{N}\right) /(N \log \log N)^{\frac{1}{2}} \stackrel{\text { a.s. }}{\rightarrow} 0
$$

as $N \rightarrow \infty$ and the result of the theorem follows from (16) and (17). 
Proof of Theorem 2. W rite $y(n)=x(n)-\mu$ and observe that for $k \geqq 1$,

$$
\begin{aligned}
y(n) y(n-k) & -E\left(y(n) y(n-k) \mid \mathscr{F}_{n-1}\right) \\
= & y(n) y(n-k)+y(n-k) \sum_{j=1}^{q} \beta(j) y(n-j) \\
= & y(n-k) \sum_{j=0}^{q} \beta(j) y(n-j)=y(n-k) \varepsilon(n),
\end{aligned}
$$

so that $\left\{\sum_{n=k+1}^{N} y(n-k) \varepsilon(n), \mathscr{F}_{N}, N>k\right\}$ is a zero mean martingale with stationary increments. Also, in view of $(2), y(n)$ is representable in the form

$$
y(n)=\sum_{j=0}^{\infty} \alpha(j) \varepsilon(n-j),
$$

where $\Sigma_{j=0}^{q} \beta(j) \alpha(n-j)=\delta_{0 n} ; \alpha(k)=0, k<0$ (e.g., Hannan [1], Chapter 1) and hence

$$
\begin{aligned}
E\left\{y^{2}(n-k) \varepsilon^{2}(n)\right\} & =E\left\{y^{2}(n-k) E\left(\varepsilon^{2}(n) \mid \mathscr{F}_{n-1}\right)\right\} \\
& =\sigma^{2} E y^{2}(n-k) \\
& =\sigma^{4} \sum_{j=0}^{\infty} \alpha^{2}(j)
\end{aligned}
$$

since $E\{\varepsilon(n-k-i) \varepsilon(n-k-j)\}=\sigma^{2} \delta_{i j}$. Furthermore,

$$
\begin{aligned}
N^{-1} \sum_{n=k+1}^{N} y^{2}(n-k) E\left(\varepsilon^{2}(n) \mid \mathscr{F}_{n-1}\right) & =\sigma^{2} N^{-1} \sum_{n=k+1}^{N} y^{2}(n-k) \text { a.s. } \\
& \stackrel{\text { a.s. }}{\rightarrow} \sigma^{4} \sum_{j=0}^{\infty} \alpha^{2}(j),
\end{aligned}
$$

using Theorem 1 of [2]. The conditions of our Theorem 1 are clearly satisfied and

$$
\begin{aligned}
& \underset{N \rightarrow \infty}{\limsup }(2 N \log \log N)^{-\frac{1}{2}} \sum_{n=k+1}^{N} y(n-k) \varepsilon(n)=\sigma^{2} c^{\frac{1}{2}} \text { a.s. } \\
& \underset{N \rightarrow \infty}{\liminf }(2 N \log \log N)^{-\frac{1}{2}} \sum_{n=k+1}^{N} y(n-k) \varepsilon(n)=-\sigma^{2} c^{\frac{1}{2}} \text { a.s. }
\end{aligned}
$$

where $c=\sum_{j=0}^{\infty} \alpha^{2}(j)$.

Now let

$$
c^{*}(j)=N^{-1} \sum_{n=1}^{N-j} y(n) y(n+j), j \geqq 0, c^{*}(-j)=c^{*}(j) .
$$


We have

$$
\sum_{n=k+1}^{N} y(n-k) \varepsilon(n)=\sum_{j=0}^{q} \beta(j) \sum_{n=k+1}^{N} y(n-k) y(n-j)
$$

$$
=N c^{*}(k)+\sum_{j=1}^{q} \beta(j)\left\{N c^{*}(k-j)-R_{N}\right\},
$$

where $R_{N}=\sum_{n=N+1}^{N+j} y(n-k) y(n-j)$. Also, since the $R_{N}$ are identically distributed (as $R$, say) with $E R^{2}<\infty$,

$$
\sum_{N} P\left(\left|R_{N}\right| \geqq \varepsilon(N \log \log N)^{\frac{1}{2}}\right) \leqq \sum_{N} P\left(|R| \geqq \varepsilon N^{\frac{1}{2}}\right)<\infty
$$

for any $\varepsilon>0$. Thus, from the Borel-Cantelli lemma,

$$
R_{N} /(N \log \log N)^{\frac{1}{2}} \stackrel{\text { a.s. }}{\rightarrow} 0
$$

as $N \rightarrow \infty$ and hence, using (19) and (20)

$$
\underset{N \rightarrow \infty}{\limsup } N^{\frac{1}{2}}(2 \log \log N)^{-\frac{1}{2}} \sum_{j=0}^{q} \beta(j) c^{*}(k-j)=\sigma^{2} c^{\frac{1}{2}} \text { a.s. }
$$

$$
\liminf _{N \rightarrow \infty} N^{\frac{1}{2}}(2 \log \log N)^{-\frac{1}{2}} \sum_{j=0}^{q} \beta(j) c^{*}(k-j)=-\sigma^{2} c^{\frac{1}{2}} \text { a.s. }
$$

Next, for any fixed $j$ we find that

$$
c^{*}(j)-c(j)=(\bar{x}-\mu)^{2}-N^{-1}\left\{\sum_{n=N-j+1}^{N} x(n)+\sum_{n=1}^{j-1} x(n)-j(\bar{x}+\mu)\right\}(\bar{x}-\mu),
$$

and with the aid of Theorem 4 it is easily seen that

$$
\lim _{N \rightarrow \infty} N^{\frac{1}{2}}(\log \log N)^{-\frac{1}{2}}\left(c^{*}(j)-c(j)\right)=0 \text { a.s., }
$$

so that using (21) and (22) we obtain

$$
\underset{N \rightarrow \infty}{\limsup } N^{\frac{1}{2}}(2 \log \log N)^{-\frac{1}{2}} \sum_{j=0}^{q} \beta(j) c(k-j)=\sigma^{2} c^{\frac{1}{2}} \text { a.s. }
$$

$$
\liminf _{N \rightarrow \infty} N^{\frac{1}{2}}(2 \log \log N)^{-\frac{1}{2}} \sum_{j=0}^{q} \beta(j) c(k-j)=-\sigma^{2} c^{\frac{1}{2}} \text { a.s. }
$$

The result of the theorem follows upon noting that, from Theorem 1 of [2], $c(0) \stackrel{\text { a.s. }}{\rightarrow} \sigma^{2} c$.

Proof of Theorem 3. First we note that $\left\{\sum_{n=1}^{N}\left(y(n) \varepsilon(n)-\sigma^{2}\right), \mathscr{F}_{N}, N \geqq 1\right\}$ is a zero mean martingale since 


$$
\begin{aligned}
E\left(y(n) \varepsilon(n) \mid \mathscr{F}_{n-1}\right) & =E\left(\varepsilon^{2}(n) \mid \mathscr{F}_{n-1}\right)+\sum_{j=1}^{\infty} \alpha(j) \varepsilon(n-j) E\left(\varepsilon(n) \mid \mathscr{F}_{n-1}\right) \\
& =\sigma^{2} .
\end{aligned}
$$

Now,

$$
\begin{array}{rl}
E\{(y(n) \varepsilon(n) & \left.\left.-\sigma^{2}\right)^{2} \mid \mathscr{F}_{n-1}\right\} \\
= & E\left(y^{2}(n) \varepsilon^{2}(n) \mid \mathscr{F}_{n-1}\right)-\sigma^{4} \\
= & \sum_{j=0}^{\infty} \sum_{k=0}^{\infty} \alpha(j) \alpha(k) E\left\{\varepsilon(n-j) \varepsilon(n-k) \varepsilon^{2}(n) \mid \mathscr{F}_{n-1}\right\}-\sigma^{4} \\
= & \sigma^{2}\left\{\sum_{j=1}^{\infty} \alpha(j) \varepsilon(n-j)\right\}^{2}+2 \sum_{j=1}^{\infty} \alpha(j) \varepsilon(n-j) E\left\{\varepsilon^{3}(n)\right\}-\sigma^{4}+\mu_{4} \\
= & \sigma^{2}\{y(n)-\varepsilon(n)\}^{2}+2 E\left\{\varepsilon^{3}(1)\right\}\{y(n)-\varepsilon(n)\}-\sigma^{4}+\mu_{4}, \\
N^{-1} \sum_{n=1}^{N} & E\left\{\left(y(n) \varepsilon(n)-\sigma^{2}\right)^{2} \mid \mathscr{F}_{n-1}\right\} \\
= & \mu_{4}-\sigma^{4}+\sigma^{2} N^{-1} \sum_{n=1}^{N}\{y(n)-\varepsilon(n)\}^{2} \\
& +2 E\left\{\varepsilon^{3}(1)\right\} N^{-1} \sum_{n=1}^{N}\{y(n)-\varepsilon(n)\} .
\end{array}
$$

Also, $\{y(n)\}$ and $\left\{E\left(y^{2}(n) \mid \mathscr{F}_{n-1}\right)\right\}$ are stationary sequences and $E y^{2}(n)<\infty$ so an application of the lemma gives

$$
N^{-1} \sum_{n=1}^{N}\left[y^{2}(n)-E\left(y^{2}(n) \mid \mathscr{F}_{n-1}\right)\right] \stackrel{\text { a.s. }}{\rightarrow} 0 .
$$

Furthermore, as already noted under weaker conditions,

and thus, from (25),

$$
N^{-1} \sum_{n=1}^{N} y^{2}(n) \stackrel{\text { a.s. }}{\rightarrow} \sigma^{2} c
$$

$$
N^{-1} \sum_{n=1}^{N} E\left(y^{2}(n) \mid \mathscr{F}_{n-1}\right) \stackrel{\text { a.s. }}{\rightarrow} \sigma^{2} c
$$

But,

and hence from (26),

$$
\begin{aligned}
E\left(y^{2}(n) \mid \mathscr{F}_{n-1}\right) & =\sum_{j=0}^{\infty} \sum_{k=0}^{\infty} \alpha(j) \alpha(k) E\left\{\varepsilon(n-j) \varepsilon(n-k) \mid \mathscr{F}_{n-1}\right\} \\
& =\{y(n)-\varepsilon(n)\}^{2}+\sigma^{2},
\end{aligned}
$$

$$
N^{-1} \sum_{n=1}^{N}\{y(n)-\varepsilon(n)\}^{2} \stackrel{\text { a.s. }}{\rightarrow} \sigma^{2}(c-1) .
$$


It then follows from (24) and (27) that

$$
N^{-1} \sum_{n=1}^{N} E\left\{\left(y(n) \varepsilon(n)-\sigma^{2}\right)^{2} \mid \mathscr{F}_{n-1}\right\}^{\text {a.s. }} \rightarrow \mu_{4}+\sigma^{4}(c-2),
$$

since $N^{-1} \sum_{n=1}^{N} y(n)=\bar{x}-\mu \stackrel{\text { a.s. }}{\rightarrow} 0$ (see Theorem 4) and $N^{-1} \Sigma_{n=1}^{N} \varepsilon(n) \stackrel{\text { a.s. }}{\rightarrow} 0$ by the strong law of large numbers. It is then easily seen that we can apply Theorem 1 and

$$
\underset{N \rightarrow \infty}{\limsup }(2 N \log \log N)^{-\frac{1}{2}} \sum_{n=1}^{N}\left(y(n) \varepsilon(n)-\sigma^{2}\right)=\left[\mu_{4}+\sigma^{4}(c-2)\right]^{\frac{1}{2}} \text { a.s. }
$$

with a corresponding result for the lim inf. The result of the theorem then follows using the same type of argument as in the proof of Theorem 2.

\section{References}

[1] Hannan, E. J. (1970) Multiple Time Series. Wiley, New York.

[2] Hannan, E. J. AND HeYDe, C. C. (1972) On limit theorems for quadratic functions of discrete time series. Ann. Math. Statist. 43, 2058-2066.

[3] Stout, W. F. (1970) The Hartman-Wintner law of the iterated logarithm for martingales. Ann. Math. Statist. 41, 2158-2160. 